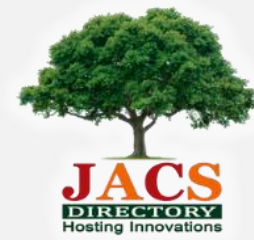

Journal of Nanoscience and Technology

\title{
Synthesis and Characterization of Chemically Deposited Nickel Sulphide Thin Film Electrodes for Electrochemical Supercapacitor Application
}

\author{
M.S. Sonawane ${ }^{1, *}$, A.M. Patil2, R.S. Patil ${ }^{3}$ \\ ${ }^{1}$ Department of Applied Science and Humanities, R.C. Patel Institute of Technology, Shirpur - 425 405, Maharashtra, India. \\ ${ }^{2}$ Department of Physics, R. C. Patel Arts, Science and Commerce College, Shirpur - 425 405, Maharashtra, India. \\ ${ }^{3}$ Department of Physics, PSGVPM's Arts, Commerce and Science College, Shahada - 425 409, Maharashtra, India.
}

\section{A R T I C L E D E T A I L S}

Article history:

Received 29 April 2019

Accepted 23 May 2019

Available online 12 June 2019

\section{Keywords:}

Nickel Sulphide Thin Films

Chemical Bath Deposition

Supercapacitor

\section{A B S T R A C T}

Nickel sulphide (NiS) thin films were synthesized on glass and stainless steel (SS) substrates using a simple chemical bath deposition route. The structural, surface morphology were characterized by X-ray diffraction (XRD) and scanning electron microscopy (SEM) respectively. The electrochemical capacitor performances were examined by using cyclic voltammetry, galvanostatic charge-discharge test and electrochemical impedance spectroscopy. In cyclic voltammetry (CV) studies, the NiS electrode exhibits the specific capacitance of $441 \mathrm{Fg}^{-1}$ has been obtained in $2 \mathrm{M} \mathrm{KOH}$ solution at a scan rate $10 \mathrm{mVs}^{-1} \mathrm{within}$ the potential range 0 to $0.8 \mathrm{~V}$ vs. $\mathrm{Ag} / \mathrm{AgCl}$. In charge-discharge behaviors, the supercapacitive parameters such as specific energy (S.E.) and specific power (S.P.) are $10.1 \mathrm{Whkg}^{-1}$ and $4.5 \mathrm{KWkg}^{-1}$ respectively. Impedance spectroscopic analysis revealed that the ESR is $11 \Omega$ in KOH electrolyte.

\section{Introduction}

Electrochemical supercapacitors are powerful energy storage systems, which have higher energy density than dielectric capacitors and have higher power density than batteries [1, 2]. Supercapacitors play an important role in various power and energy source applications [3]. Supercapacitors are classified into two types based on charge storage mechanisms, electrical double-layer capacitors dominated by electrostatic charge diffusion and accumulation at the interface of the electrode/ electrolyte and pseudocapacitors governed by Faradaic reactions at the electrode materials [4-6].

Supercapacitor electrode materials have three types such as carbon, metal oxides and polymers. Among the electroactive materials, $\mathrm{RuO}_{2}$ possesses pseudocapacitance property and exhibits much higher specific capacitance, highly reversible redox reaction and excellent cycle life [7-9]. However, the high cost and toxic nature of $\mathrm{RuO}_{2}$ has prompted the search for other transition metal oxides/hydroxides [4]. In the last few years, metal sulfides have recently emerged as a new type of electroactive materials due to their excellent redox reversibility and relatively high capacity/capacitance [10-13]. We have chosen NiS as an electrode for supercapacitor because of its higher stability, high abundance, low cost, better safety, and environmental friendliness in comparison with other metal sulphide. Many techniques have been currently used in order to produce NiS thin films electrode. Ting Zhu et al. synthesized hierarchical NiS hollow spheres by an efficient template-engaged conversion method. Researchers found that, NiS hollow spheres exhibit high specific capacitances of 583-927 $\mathrm{Fg}^{-1}$ at various current densities of 4.08-10.2 $\mathrm{Ag}^{-1}$ [14]. Jiaqin Yang et al. studied the novel hierarchical flower-like nickel sulfide assembled by nanoplates has been successfully synthesized via a template-free and one-step solvothermal method. Researchers investigated that the sample with more nanoplates shows a higher specific capacitance and promising cycling stability. After 3000 cycles, a high specific capacitance of $778.8 \mathrm{Fg}^{-1}$ maintains at current density is $4 \mathrm{Ag}^{-1}$ [15]. Apostolova et al. reported a thin-layer $\mathrm{NiS}$ electrode is capable of reversible electrochemical transformation during more than 200 cycles with an output capacitance of $143 \mathrm{Fg}^{-1}$ [16]. Among these deposition techniques, chemical bath deposition presently attracts considerable attention, as it does not require sophisticated instrument. Any insoluble

*Corresponding Author:milindkr.2000@gmail.com(M.S. Sonawane) 
PARSTAT 4000). A typical three electrode cell was employed; NiS as a working electrode platinum wire as the counter electrode, and $\mathrm{Ag} / \mathrm{AgCl}$ used as the reference electrode. All electrochemical experiments were carried out at room temperature.

\section{Results and Discussion}

\subsection{Structural and Morphological Analysis}

The morphology and crystal structure of nanocrystalline NiS thin films were investigated by scanning electron microscopy and X-ray diffraction techniques respectively. Structural analysis of NiS thin films was carried out by X-ray diffraction technique. Fig. 1 shows typical XRD pattern of NiS thin film on glass substrate. Nanocrystalline nature of NiS films is confirmed from XRD pattern since observed diffraction peaks are weak and are of low intensity. Comparison of $d$-values with JCPDS 02-1280 and 86-2280 data for NiS shows that the material is NiS having hexagonal and rhombohedral structure. The NiS thin film having four diffraction peaks at angles $2 \theta \sim 37.42^{\circ}, 46.57^{\circ}, 60.68^{\circ}$ and $81.62^{\circ}$ are correspond to (220), (102), (103) and (161) plane respectively [19-21]. It is observed that, NiS film is prepared at room temperature having grain size is $\sim 21 \mathrm{~nm}$.

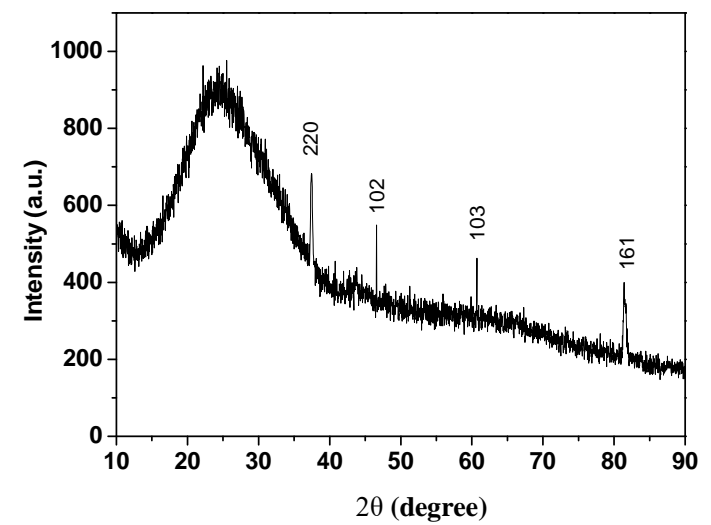

Fig. 1 XRD pattern of NiS thin films

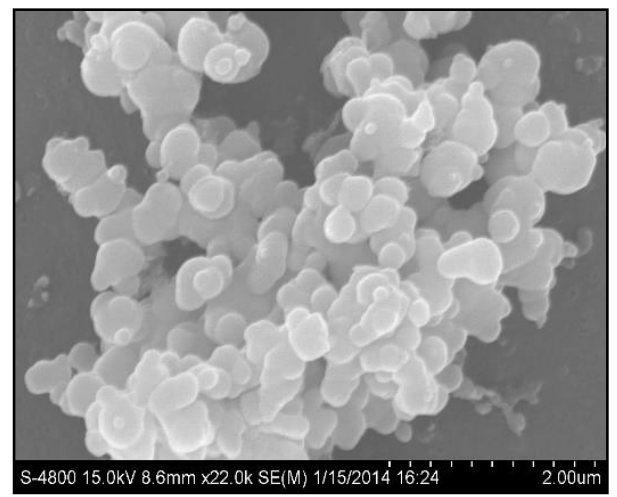

Fig. 2 The SEM image of as-deposited NiS film

Fig. 2 shows the SEM image of the MCBD deposited NiS thin film. It is observed from the micrographs that NiS film is homogeneous, fine grained and well covered to the substrate with overgrowth of some particles. The overall surface structure is seen to have grains of spherical shape. These films revealed that grains were very small in size with no well-defined grain boundaries. The average grain size of NiS film is found that $300 \mathrm{~nm}$. Some micro porous space between the fine particles can also be seen. The porosity of films can enhance the redox [20].

\subsection{Supercapacitive Properties of the NiS Thin Film Electrode}

Chemically deposited NiS electrodes were used in the electrochemical capacitor and their performances were tested using CV. To perform cyclic voltammetry tests a series of changing voltages at a constant sweep rate $(\mathrm{dV} / \mathrm{dt})$ is applied and the response current is recorded. The supercapacitive studies carried out by means of effect of scan rate and stability studies. The capacitance can be estimated by the following equation $[6,22]$,

$$
C\left(V_{f}-V_{i}\right)=q=\frac{1}{v} \int_{V_{i}}^{V_{f}} I(V) d V
$$

where, $C$ is the total capacitance, I the current density $\left(A / \mathrm{cm}^{2}\right), v$ the sweep rate $(\mathrm{V} / \mathrm{s}), v_{i}$ the initial and $v_{f}$ the final voltages $(\mathrm{V})$. The integral on the right-hand side of Eq.(1) is the area under the CV. Thus, the total surface charge, (or total capacitance) of the deposit material can be estimated by evaluating the area under the capacitive current-voltage curve portion of a CV. The specific capacitance $\left(\mathrm{Fg}^{-1}\right)$ of the electrode was obtained by dividing the capacitance to weight dipped in the electrolyte. The interfacial capacitance $\left(\mathrm{Fcm}^{-2}\right)$ was obtained by dividing the capacitance to area dipped in the electrolyte.

Cyclic voltamogram of the NiS electrode of thickness $0.00016 \mathrm{~g} / \mathrm{cm}^{2}$, in aqueous electrolyte $2 \mathrm{M}$ solution of $\mathrm{KOH}$ were studied in the voltage range of 0 to $0.8 \mathrm{~V} \mathrm{Vs} \mathrm{Ag/AgCl}$. The $\mathrm{KOH}$ electrolyte gave the largest current, which was greater than the other electrolytes. The area of working electrode was $1 \mathrm{~cm}^{2}$. The voltammetric responses of NiS electrode at different scan rates are shown in Fig. 3. It was found that the current under curve is slowly increased with scan rate. This showed that voltammetric currents are directly proportional to the scan rate of $\mathrm{CV}$, indicating an ideally capacitive behavior [14-16,23-25].

Variation of specific capacitance and interfacial capacitance values with scan rate is shown in Fig. 4. The specific and interfacial capacitance values are decreased from 441 to $160 \mathrm{Fg}^{-1}$ and 0.071 to $0.026 \mathrm{Fcm}^{-2}$ respectively, as the scan rate was increased from 10 to $100 \mathrm{mVs}^{-1}$. As the scan rate is increased, there is a slight decrease in the specific capacitance that is comparable to previously reported data. At lowest scan rate $10 \mathrm{mVs}^{-1}$ maximum capacitance is found to be $441 \mathrm{Fg}^{-1}$.

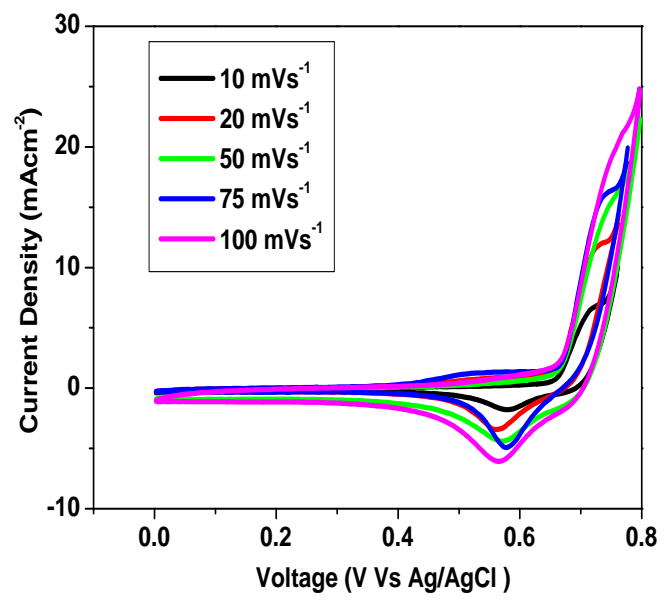

Fig. 3 The CV curves of NiS electrode at 10 to $100 \mathrm{mVs}^{-1}$ scan rates in $2 \mathrm{M} \mathrm{KOH}$ electrolyte

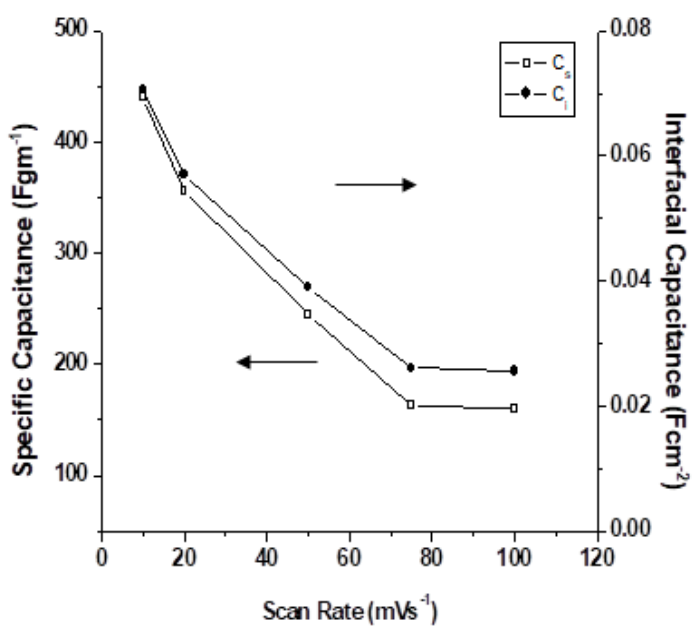

Fig. 4 The specific and interfacial capacitances of NiS electrode at 10 to $100 \mathrm{mVs}^{-1}$ scan rates in $2 \mathrm{M} \mathrm{KOH}$ electrolyte

\subsection{Galvanostatic Charge-Discharge Studies}

The electrodes of NiS were subjected to galvanostatic charge-discharge cycling between 0 and $0.8 \mathrm{~V}$ in $2 \mathrm{M} \mathrm{KOH}$ solution at different current density of $0.5,1$ and $2 \mathrm{mAcm}^{-2}$. Typical curves of potential variation with time of cycling are shown in Fig. 5, it can be seen that, the nonsymmetric behavior of voltage-time curve was seen, that is IR drop was observed. The discharge profile showed two parts; a resistive component arising from the sudden voltage drop (linear portion parallel to y-axis) representing the voltage change due to the internal resistance and a capacitive component 
(curved portion) related to the voltage change due to the change in energy within the capacitor. The specific energy (E), and specific power (P) and coulomb efficiency $(\eta \%)$ are calculated using following equations $[4,6,26]$,

$$
\begin{aligned}
& \mathrm{E}=\left(\frac{0.5}{3.6}\right) \mathrm{C}_{\mathrm{s}}(\Delta \mathrm{V})^{2} \\
& \mathrm{P}=\left(\frac{E}{t_{d}}\right)
\end{aligned}
$$

And, Columbic efficiency (\%) $\eta=\left(\frac{t_{d}}{t_{c}}\right) \times 100$

where, $C_{s}$ is the specific capacitance and $\Delta V(\mathrm{~V})$ is the voltage window. $t_{d}$ is discharging time $(\mathrm{s}), t_{c}$ is charging time (s).

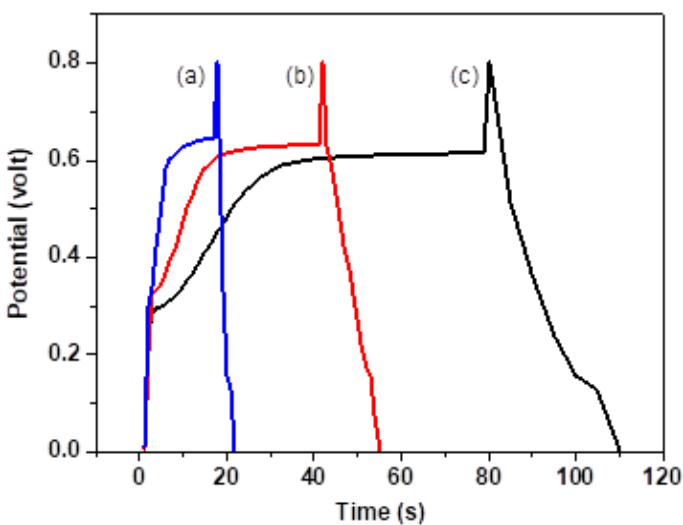

Fig. 5 Charging-discharging behavior of NiS electrode at a current density of (a) 2, (b) 1 , (c) $0.5 \mathrm{mAcm}^{-2}$ in $2 \mathrm{M} \mathrm{KOH}$ electrolyte

The capacitance, energy density and power density of the NiS electrode were calculated using Eqs.(2-4). The specific capacitance value is 113,94 and $47 \mathrm{Fg}^{-1}$ for NiS thin film electrode at current density $0.5,1$ and 2 $\mathrm{mAcm}^{-2}$, respectively. The maximum energy density of $10.1 \mathrm{Whkg}^{-1}$ and power density of $4.5 \mathrm{kWkg}^{-1}$ were obtained at a current density 0.5 $\mathrm{mA} / \mathrm{cm}^{2}$. This increase in specific energy and power may be due to increased time for charging and discharging of capacitor. The energy efficiency, 36\%, was obtained. Variation of energy density with power density values of NiS electrode at different current densities is shown in Fig. 6.

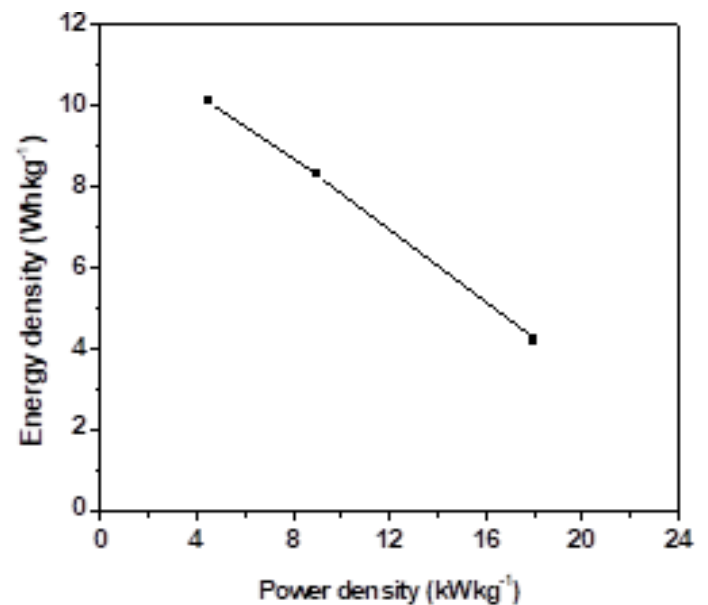

Fig. 6 Variation of energy density with power density values of NiS electrode at different current densities

\subsection{Electrochemical Impedance Analysis (EIS Studies)}

Electrochemical impedance spectroscopy, a powerful technique for the investigation of the capacitive behavior of electrochemical cells, has been also used to characterize our materials.

Nyquist plots obtained for as deposited $\mathrm{NiS}$ electrodes at $0.8 \mathrm{~V}$ Vs $\mathrm{Ag} / \mathrm{AgCl}$ is shown in Fig. 7, where $\mathrm{Z}_{\mathrm{re}}{ }^{\prime}$ and $\mathrm{Z}_{\mathrm{im}}{ }^{\prime \prime}$ are the real and imaginary parts of the impedance, respectively. It displays a semicircle in the high frequency and a linear curve in the low-frequency region. The semicircle in the high-frequency region is related to the reaction kinetics at the electrode and electrolyte interfaces. The linear curve at the low-frequency region can be attributed to the diffusion-controlled process in the electrolyte [15, 24, 25]. The initial non-zero intercepts in high frequency regime at the beginning of the semicircle and is due to the electrical resistance of the electrolyte $\left(\mathrm{R}_{\text {ele }}\right)$. The value for ESR for as deposited NiS a thin film is $11 \Omega$ in $\mathrm{KOH}$ electrolyte. https://doi.org/10.30799/jnst.243.19050405

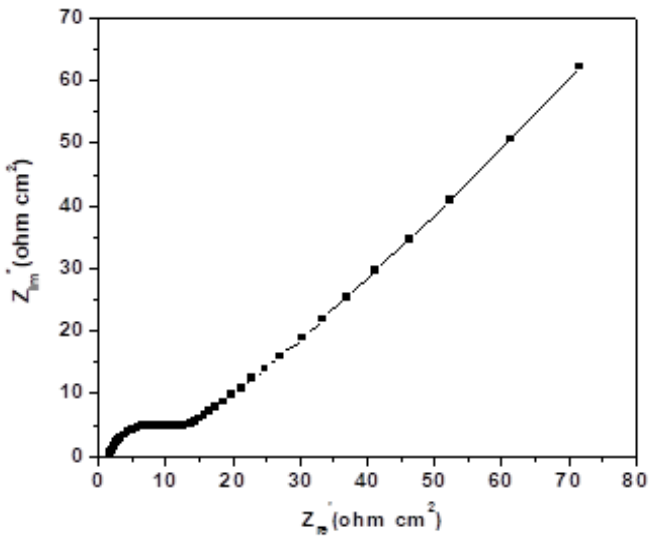

Fig. 7 Nyquist plots of nickel sulphide electrode in $2 \mathrm{M} \mathrm{KOH}$

\section{Conclusion}

In conclusion, NiS thin film electrodes have been prepared successfully at room temperature by chemical bath deposition method and used in supercapacitors. The XRD analysis showed the NiS thin films are amorphous. The SEM image of NiS film showed porous structure and the film surface covered with agglomerates of different sizes. The as-prepared NiS thin film electrode has excellent electrochemical capacitive characteristic with potential range 0 to $+0.8 \mathrm{~V} \mathrm{Vs} \mathrm{Ag} / \mathrm{AgCl}$ at constant concentration $2 \mathrm{M} \mathrm{KOH}$ solution. The electrochemical study revealed that the NiS electrode showed high specific capacitance of $441 \mathrm{Fg}^{-1}$. Chargedischarge curves confirmed that the capacitance consisted from EDLC and psedudocapacitance. The specific energy (E), specific power (P) and coulomb efficiency $(\eta \%)$ was $10.1 \mathrm{Whkg}^{-1}$ and $4.5 \mathrm{kWkg}^{-1}$, and $36 \%$, respectively. Impedance spectroscopic analysis revealed that the ESR is $11 \Omega$ in KOH electrolyte.

\section{Acknowledgement}

The authors would like to thank the Department of Physical Sciences, NMU, Jalgaon and Nano Materials and Device Laboratory, Applied Physics Department, VNIT, Nagpur for the provision of characterization facilities. Authors are thankful to Principal, PSGVPM'S ASC College, Shahada and Principal, R.C. Patel Institute of Technology, Shirpur for constant support throughout this work.

\section{References}

[1] R. Kotz, M. Carlen, Principles and applications of electrochemical capacitors Electrochim. Acta 45 (2000) 2483-2498.

[2] H.Z. Wan, J.J. Jiang, J.W. Yu, Y.J. Ruan, L. Peng, et al., Cobalt sulfide nanotube arrays grown on FTO and graphene membranes for high-performance supercapacitor application, Appl. Surf. Sci. 311 (2014) 793-798.

[3] A.I. Inamdar, Y.S. Kim, J.S. Sohn, H. Im, Supercapacitive characteristics of electrodeposited polyaniline thin films grown on indium-doped tin-oxide substrates, Jour. Korean Phys. Soc. 59 (2011)145-149.

[4] Fulian Luo, Jing Li, Hongyan Yuan, Dan Xia, Rapid synthesis of threedimensional flower-like cobalt sulfide hierarchitectures by microwave assisted heating method for high-performance supercapacitors, Electrochim. Acta 123 (2014) 183-189.

[5] C.D. Lokhande D.P. Dubal, Oh-Shim Joo, Review metal oxide thin film-based supercapacitors, Curr. Appl. Phys. 11 (2011) 255-270.

[6] S.M. Chen, R. Ramachandran, V. Mani, R. Saraswathi, Recent advancements in electrode materials for the high-performance electrochemical supercapacitors: A review, Int. J. Electrochem. Sci. 9 (2014) 4072-4085.

[7] Yan-Yu Liang, Hu Lin Li, Xiao-Gang Zhang, Solid state synthesis of hydrous ruthenium oxide for supercapacitors, J. Power Sources 173 (2007) 599-605.

[8] P. Justin, G. Ranga Rao, CoS spheres for high-rate electrochemical capacitive energy storage application, Int. J. Hyd. Energy 35 (2010) 9709-9715.

[9] J.P. Zheng, P.J. Cygan, T.R. Jow, Hydrous ruthenium oxide as an electrode material for electrochemical capacitors, J. Electrochem. Soc. 142 (1995) 26992703.

[10] P. Kulkarni, S.K. Nataraj, R. Geetha Balakrishna, D.H. Nagaraju, M.V. Reddy, Nanostructured binary and ternary metal sulfides: synthesis methods and their application in energy conversion and storage devices, J. Mater. Chem. A 5 (2017) 22040-22094.

[11] S.J. Bao, C.M. Li, C.X. Guo, Y. Qiao, Biomolecule-assisted synthesis of cobalt sulfide nanowires for application in supercapacitors, J. Power Sources 180 (2008) 676-681.

[12] Q. Wang, L. Jiao, H. Du, J. Yang, Q. Huan, et al., Facile synthesis and superior supercapacitor performances of three-dimensional cobalt sulfide hierarchitectures, Cryst. Eng. Comm. 13 (2011) 6960-6963. 
[13] F. Tao, Y.Q. Zhao, G.Q. Zhang, H.L. Li, Electrochemical characterization on cobalt sulfide for electrochemical supercapacitors, Electrochem. Commun. 9 (2007) 1282-1287.

[14] T. Zhu, Z. Wang, S.Ding, J.S. Chen, X. Wen (David) Lou, Hierarchical nickel sulfide hollow spheres for high performance supercapacitors, RSC Adv. 1 (2011) 397 400 .

[15] J. Yang, W. Guo, D. Li, C. Wei, H. Fan, et al., Synthesis and electrochemical performances of novel hierarchical flower-like nickel sulfide with tunable number of composed nanoplates, J. Power Sources 268 (2014) 113-120.

[16] R.D. Apostolova, O.V. Kolomoyets, Yu. A. Tkachenko, E.M. Shembel, Electrolytic Nickel Sulfide in a Model Electrochemical Capacitor, Rus. J. Appl. Chem. 85 (2012) 612-615.

[17] R.S. Mane, C.D. Lokhande, Review chemical deposition method for metal chalcogenide thin films, Mater. Chem. Phys. 65 (2000) 1-31.

[18] K.L. Chopra, Thin film phenomena, Mc Graw Hill Book Co., New York, 1996.

[19] P. Pramanik, S.J. Biswas, Deposition of nickel chalcogenide thin film by solution grow techniques, Solid State Chem. 65 (1986) 145-147.
[20] A. Kassim, H.S. Min, T.W. Tee, N.C. Fei, Influence of triethanolamine on the chemical bath deposited NiS thin films, Am. J. Appl. Sci. 8 (2011) 359-361.

[21] F. Atay, S. Kose, V. Bilgin, I. Akyuz, Electrical, optical, structural and morphological properties of NiS films, Turk. J. Phys. 27 (2003) 285-291.

[22] E.E. Kalu, T.T. Nwoga, V. Srinivasan, J.W. Weidner, Cyclic voltammetric studies of the effects of time and temperature on the capacitance of electrochemically deposited nickel hydroxide, J. Power Sources 92 (2001) 163-167.

[23] X. Yan, X. Tong, L. Ma, Y. Tian, Y. Cai, et al., Synthesis of porous NiS nanoflake arrays by ion exchange reaction from $\mathrm{NiO}$ and their high-performance supercapacitor properties, Mater. Lett. 124 (2014) 133-136.

[24] Y. Li, K. Ye, K. Cheng, J. Yin, D. Cao, G. Wang, Electrodeposition of nickel sulfide on graphene-covered make-up cotton as a flexible electrode material for highperformance supercapacitors, J. Power Sources 274 (2015) 943-950.

[25] S.W. Chou, J.Y. Lin, Cathodic deposition of flaky nickel sulfide nanostructure as an electroactive material for high-performance supercapacitors, J. Electrochem. Soc. 160(4) (2013) D178-D182.

[26] Z. Yang, C.Y. Chen, H.T. Chang, Supercapacitors incorporating hollow cobalt sulfide hexagonal nanosheets, J. Power Sources 196 (2011) 7874-7877. 